\title{
Computed tomography-3D-volumetry: a valuable adjunctive diagnostic tool after bariatric surgery
}

\author{
Christine Stier ${ }^{1}$, Chetan Parmar ${ }^{2}$, Ann-Cathrin Koschker ${ }^{3}$, Mohammed Bokhari $^{4}$, Raphael Stier ${ }^{5}$, Sonja \\ Chiappetta $^{6}$ \\ 'Bariatric and Metabolic Surgery, Obesity Centre NRW, Sana Hospitals Germany, Huerth 50354, Germany. \\ ${ }^{2}$ Bariatric and Metabolic Surgery, The Whittington Hospital NHS Trust, London N19 5NF, UK. \\ ${ }^{3}$ Department of Internal Medicine, Division of Endocrinology, University Hospital, University of Würzburg, Würzburg 97080, \\ Germany. \\ ${ }^{4}$ Department of Radiology, Sana Klinikum Offenbach, Offenbach 63069, Germany. \\ ${ }^{5}$ Department of General, Visceral and Cancer Surgery, University of Cologne, Cologne 50937, Germany. \\ ${ }^{6}$ Bariatric and Metabolic Surgery, Ospedale Evangelico Betania, Napoli 80147, Italy.
}

Correspondence to: Dr. Christine Stier, Bariatric and Metabolic Surgery, Obesity Centre NRW, Sana Hospitals Germany, Huerth 50354, Germany. E-mail: christine.stier@sana.de

How to cite this article: Stier C, Parmar C, Koschker AC, Bokhari M, Stier R, Chiappetta S. Computed tomography-3Dvolumetry: a valuable adjunctive diagnostic tool after bariatric surgery. Mini-invasive Surg 2020;4:18. http://dx.doi.org/10.20517/2574-1225.2019.75

Received: 31 Dec 2019 First Decision: 17 Feb 2020 Revised: 25 Feb 2020 Accepted: 2 Mar 2020 Published: 11 Mar 2020

Science Editor: Wah Yang Copy Editor: Jing-Wen Zhang Production Editor: Tian Zhang

\begin{abstract}
Aim: After bariatric surgery, a variety of complaints may arise. Identification of the causes of such symptoms is often challenging due to the postoperatively modified anatomy. While standard examinations with upper endoscopy and upper gastrointestinal series might miss the three-dimensional anatomic nature of the problem, quantitative three-dimensional computed tomography volumetry (3D-CT) of the upper gastrointestinal tract offers a novel, adjunctive examination, revealing the detailed anatomy. The aim of this study was to analyse the clinical value of 3D-CT in post-bariatric patients.
\end{abstract}

Methods: Prospective data of 279 patients, who underwent 3D-CT due to complications after different bariatric procedures, were retrospectively analysed. Directly before examination, the surgical-modified stomach was distended with an effervescent-powder. CT images were 3D-reconstructed and, further, gastric volume was calculated.

Results: In total, 279 patients were examined. Time between surgery and examination was significantly different between Roux-en-Y gastric bypass ( $n=168)(54.3 \pm 38.6$ months) and sleeve gastrectomy $(n=78)(27.8 \pm 21.7$ months) ( $P=0.0001)$. Others, less numerous, but included procedures were one-anastomosis/mini gastric bypass $(n=11)$, and dated procedures, such as the vertical banded gastrostomy. The examination allowed calculation of the gastric volume, and the 3D-reconstructions depicted accurately the pivotable anatomic details of the modified upper gastrointestinal tract with $360^{\circ}$ view. As a robust result, patients with a higher gastric volume showed more weight regain after sleeve gastrectomy.

cc) (i) The Author(s) 2020. Open Access This article is licensed under a Creative Commons Attribution 4.0 International License (https://creativecommons.org/licenses/by/4.0/), which permits unrestricted use sharing, adaptation, distribution and reproduction in any medium or format, for any purpose, even commercially, as long as you give appropriate credit to the original author(s) and the source, provide a link to the Creative Commons license, and indicate if changes were made. 
Conclusion: 3D-CT is easy-to-perform and facilitates identification of the post-surgical three-dimensional gastric anatomy. It represents a valuable additional diagnostic tool in post-bariatric patients with post-procedural complications. 3D-CT might be an important preoperative tool prior to revisional surgery. In addition, this is the only exact and reproducible calculation of the gastric volume.

Keywords: Gastrointestinal tract, computed tomography, gastric volumetry, 3D-reconstruction, anatomical accuracy

\section{INTRODUCTION}

Bariatric surgery has been shown to be an effective and safe treatment for obesity and metabolic disorders ${ }^{[1-3]}$. However, a number of postsurgical complications may arise, including gastroesophageal reflux disease (GERD), epigastric pain, vomiting, and, especially in bariatric patients, poor weight loss or weight regain ${ }^{[4,5]}$. These symptoms can appear at different times. Some are evident already shortly after the operation, but others appear only years after a procedure. Whilst weight regain is such a special issue in bariatric patients, an objective measurement of gastric or pouch volume is difficult, even if the increase in gastric volume is being discussed more and more as an underlying cause. Comparability of former, contemporary and future examinations is even more complex. Upper endoscopy (UE) and upper gastrointestinal series (UGI) are the most important diagnostic tools after general surgery of the upper gastrointestinal tract. While the sensitivity of UE is high in patients with upper gastrointestinal symptoms $^{[6]}$, in the case of insufficient weight loss or weight regain, as well as in anatomically confusing conditions, UE and UGI contrast studies are often not conclusive. UE is more useful in gathering information concerning pouch- or stoma-related complications, whereas UGI is a more effective means of detecting oesophageal or Roux-limb abnormalities ${ }^{[7]}$, all of which are possible standard reasons for upper abdominal pain after bariatric surgery, but mostly do not account for an occurring weight regain or, moreover, the special complications that have already tried to be clarified before and elsewhere.

Quantitative three-dimensional computed tomography volumetry (3D-CT) of the upper gastrointestinal tract is a not very widespread technique and is rarely used in general, but for years has been frequently used in some specialised bariatric centres. By providing pivotable, 3D-reconstructed pictures of the anatomy, on the one hand, it enables robust and accurate preoperative planning in patients undergoing complex revisional bariatric surgery ${ }^{[8]}$. On the other hand, and as a worthwhile side-effect of the technique itself, 3D-CT is a useful and exclusive tool for accurate volume measurement. For example, with the aid of $3 \mathrm{D}-\mathrm{CT}$, Hanssen et al. ${ }^{[9]}$ demonstrated that initial sleeve volume $\geq 100 \mathrm{~mL}$ is significantly related to insufficient weight loss after bariatric surgery.

The aim of this study was to demonstrate the clinical usefulness of 3D-CT to assess accurately the shape and anatomy, and, further, its additional value as an exclusive diagnostic tool for gastric volumetry and quantitation after bariatric surgery.

\section{METHODS}

\section{Examination protocol of the 3D-CT}

To achieve a high level of comparability, a standardised examination protocol for the 3D-CT was invented and established years ago. A dedicated and trained bariatric team monitored all examinations.

Patients had to fast at least $6 \mathrm{~h}$ before the scheduled examination. To prepare for the CT-study, shortly before the examination, all patients received $20 \mathrm{mg}$ butylscopolamin as intravenous injection to reduce gastrointestinal motility during the procedure. Immediately before scanning, each patient swallowed $11.8 \mathrm{~g}$ (two sachets) of a commercially available effervescent powder (Ahoi Brause, Frigeo), which is normally used in the preparation 
of an aromatised, acidulous sherbet or just as sour candy. A contrast itself is not necessary for the examination. Patients were already sitting on the CT-table during the intake. The effervescent powder creates an instant froth, thus causing immediate distention of the stomach and its adjacent anatomical structures during examination. In addition, the patients were instructed to keep the froth strictly within the stomach and therefore avoid belching. Another reason for insufficient distention can be a prolonged time span between intake and examination. Thus, immediately after intake, the patient lies back to a supine examination position. Directly afterwards, the images are acquired using a Philips Brilliance 64-slice CTscanner. The scan itself is recorded with a collimation of $32 \mathrm{~mm} \times 1.25 \mathrm{~mm}$. This defines the table traverse speed during one gantry rotation of $32 \mathrm{~mm}$, thus capturing a $1.25 \mathrm{~mm}$ layer. The corresponding pitch factor is 0.906 . After the examination, the $3 \mathrm{D}$ - reconstruction is calculated with the Philips workstation and the IntelliSpace Portal. The resulting 3D-pictures are $360^{\circ}$ rotatable, and accurately display the stomach and its adjacent gastrointestinal structures, here integrated in the patient's semi lucent skeleton.

\section{Statistics}

During 24 months, 279 patients underwent the 3D-CT at Sana Klinikum Offenbach, a high-volume certified centre of excellence for obesity and metabolic surgery by the European Accreditation Council for Bariatric Surgery, as part of our standard diagnostic algorithm for patients after bariatric surgery with remaining unclear symptoms after standard diagnostic examinations (UE and UGI). Patients with various bariatric procedures were included [sleeve gastrectomy (SG), Roux-en-Y gastric bypass (RYGB), oneanastomosis/mini gastric bypass (OAGB/MGB), gastric banding (GB), vertical banded gastrostomy (VBG) and biliopancreatic diversion (BPD)], which led to the definition of three main subgroups (Bypass, SG and others). Examination data were collected prospectively and evaluated retrospectively.

Demographic and clinical data include gender, age, height in $\mathrm{cm}$, weight in $\mathrm{kg}, \mathrm{BMI}$ in $\mathrm{kg} / \mathrm{m}^{2}$ prior to surgery and prior to the examination and excess weight loss (EWL) in assuming ideal body weight to be that equivalent to a BMI of $25 \mathrm{~kg} / \mathrm{m}^{2}$. Time between surgery and examination was considered. Statistical analysis was performed using SPSS 11.0 statistical software for Microsoft Windows (SPSS Inc., Chicago, IL, USA). Continuous variables, when normally distributed, were reported as mean, standard deviation (SD) and range. Intergroup differences were tested by a two-sample $t$ test for normally distributed data. A $P$-value $<0.05$ was considered significant.

The study was conducted in accordance with the principles of the Declaration of Helsinki. This analysis represents a partial result of a study, evaluating postsurgical endoscopies within this period, which was reviewed and approved by the ethics committee of the regional regulatory institution, Landesärztekammer Hessen (FF 111/2016; ClinicalTrials.gov Identifier: NCT03532646). Additionally, all participants provided written informed consent for data sharing.

\section{RESULTS}

\section{Descriptive statistics}

\section{General patient data}

This study included 279 post-bariatric patients [Table 1], of whom 223 were females (79.9\%). Only some of the patients came from the centre's primary collection. Nearly $37 \%(103 / 279,36.91 \%)$ were referred from other national or international bariatric centres, with the treatment mandate to solve complications that were previously intractable.

Significantly more than half of the patients $(183 / 279 ; 65.6 \%)$ underwent a bypass procedure (proximal Roux-en-Y, $n=168$, and OAGB/MGB, $n=15)$ and 74 patients (26.5\%) had a sleeve gastrectomy. Patients' data are shown in Table 1. 
Table 1. Patient data

\begin{tabular}{|c|c|c|c|c|c|}
\hline & RYGB & SG & OAGB/MGB & BPD-DS/BPD/SADI-S & GB VBG \\
\hline n 279 (F223/M56) & 168 (F142/M26) & 78 (F56/M22) & $11(F 7 / M 4)$ & $7(\mathrm{~F} 7 / \mathrm{MO})$ & 15 (F13/M2) \\
\hline Age $(y)$ & $43.88 \pm 10.87$ & $45.84 \pm 11.11$ & $46.09 \pm 9.48$ & $45.86 \pm 9.84$ & $46.80 \pm 9.26$ \\
\hline Height $(\mathrm{cm})$ & $166.84 \pm 7.93$ & $169.91 \pm 10.04$ & $170.14 \pm 8.11$ & $166.57 \pm 6.65$ & $166.73 \pm 9.28$ \\
\hline Weight at surgery (kg) & $140.88 \pm 26.91$ & $159.35 \pm 30.58$ & $165.45 \pm 29.97$ & $172.00 \pm 34.08$ & $152.93 \pm 35.78$ \\
\hline Weight at examination $(\mathrm{kg})$ & $100.03 \pm 26.64$ & $120.15 \pm 34.26$ & $112.89 \pm 34.09$ & $108.91 \pm 15.91$ & $126.20 \pm 25.42$ \\
\hline $\mathrm{BMI}\left(\mathrm{kg} / \mathrm{m}^{2}\right)$ at surgery & $50.68 \pm 9.02$ & $55.14 \pm 9.12$ & $57.09 \pm 8.75$ & $61.61 \pm 8.87$ & $54.85 \pm 12.16$ \\
\hline Excess weight at surgery $(\mathrm{kg})$ & $74.0 \pm 25.0$ & $89.45 \pm 26.53$ & $95.32 \pm 26.94$ & $105.43 \pm 29.38$ & $86.20 \pm 32.52$ \\
\hline EWL (\%) & $57.27 \pm 29.28$ & $46.26 \pm 26.35$ & $56.12 \pm 31.95$ & $55.11 \pm 23.89$ & $22.00 \pm 45.69$ \\
\hline TBWL (kg) & $40.92 \pm 21.87$ & $39.20 \pm 23.44$ & $52.56 \pm 33.07$ & $63.09 \pm 43.40$ & $26.73 \pm 27.12$ \\
\hline TBWL (\%) & $28.75 \pm 14.20$ & $24.95 \pm 13.70$ & $31.18 \pm 17.81$ & $34.11 \pm 16.93$ & $15.07 \pm 18.69$ \\
\hline Time-elapse from surgery to examination (months) & $54.28 \pm 38.54$ & $27.78 \pm 21.71$ & $16.45 \pm 15.46$ & $42.14 \pm 17.24$ & $173 \pm 52.71$ \\
\hline
\end{tabular}

RYGB: roux-en-Y gastric bypass; SG: sleeve gastrectomy; BPD: biliopancreatic diversion; GB: gastric banding; VBG: vertical banded gastrostomy; EWL: excess weight loss; BMI: body mass index; OAGB/MGB: one-anastomosis/mini gastric bypass; Sadi-S: Single anastmosis duodeno-ileal bypass with sleeve gastrectomy; TBWL: total body weight loss; BPD-DS: biliopancreatic diversion with duodenal switch

\section{Procedure data}

The medium time from primary surgery to introduction was $51.34 \pm 46.85$ months in the overall cohort ( $n$ = 279). Eleven patients $(n=11)$ presented with rarer and more dated procedures, including GB and VBG. In those patients, time between surgery and re-evaluation due to complaints was $173.20 \pm 52.71$ months. All those bariatric procedures other than RYGB or SG were combined and added to this third subgroup, including GB, VBG (together $n=15)$, BPD $(n=7)$ and OAGB/MGB $(n=11)$ procedures (total $n=33)$. Analysing the other two main subgroups - RYGB and SG - demonstrated a highly significant difference in the time between surgery and reported complaints. Time span to the actual reported emergency-evaluation was $54.3 \pm 38.6$ months after a RYGB and $27.8 \pm 21.7$ months after SG $(P=0.0001)$.

\section{Complaints - weight regain}

The vast majority of patients reported non-specific worsening abdominal pain, which was the most common indication for examination. However, a closer exploration often revealed the most feared patient concern, which is weight regain $(49.82 \% ; 139 / 276)$ regardless of the severity of the existing complaints. This additionally affected 61 patients after SG (78.20\% 61/78) and 65 patients after RYGB (38.7\%; 65/168). The medium gastric volume of the 3D volumetry was $174.41 \pm 59.36 \mathrm{~mL}$ in SG and $47.91 \pm 20.86 \mathrm{~mL}$ in RYGB. The Pearson's chi-square value was calculated for all SG volumes and the contemporarily related EWL. A bilateral signification of $0.005,(P<0.01)$ as inverse relation was found between volume and EWL with a confidence level of $99 \%$.

\section{GERD and hiatal hernia}

GERD was another frequently reported symptom, which affected predominantly patients with SG (39/78; $50 \%$ ), VBG, GB and BPD (in total, 52/279; 18.63\%). After sleeve gastrectomy, 3D-CT revealed in $47.29 \%$ (35/74) a hiatal hernia, whereas, following RYGB, hiatal hernias were detected only in $16.07 \%$ (27/168). It is noteworthy that there was no significant difference in the detection rate or the longitudinal quantitation of a hiatal hernia, when the results of endoscopic examination and 3D-CT were compared $(2.55 \pm 0.82 \mathrm{~cm} v s .2 .24$ $\pm 1.13 \mathrm{~cm}$ in RYGB and $3.04 \pm 1.23$ vs. $2.69 \pm 1.59$ in SG). However, especially in difficult cases, the detailed imaged anatomy showed more details, which were easier to reveal, and therefore provided additional and often therapy-critical information. It directly influenced the objectivity of findings and, thus, the decisionmaking security. Due to the additional information resulting from $3 \mathrm{D}-\mathrm{CT}$, which revealed a twisting, relative constriction or a remnant and herniated part of the fundus after SG, 12 of the patients underwent directly conversion to RYGB without previous conservative therapeutic attempt. The major finding was that $3 \mathrm{D}$-CT had direct impact on the resulting patient treatment in more than $21 \%$ of cases, without performing another UGI, which had already previously been carried out without success in the referring departments. 
Table 2. Results and comparison of upper endoscopy and 3D-CT

\begin{tabular}{|c|c|c|c|c|}
\hline & RYGB UE & RYGB 3D-CT & SG UE & SG 3D-CT \\
\hline Hiatal hernia Longitudinal measure $(\mathrm{cm})$ & $2.55 \pm 0.82$ & $2.24 \pm 1.13$ & $3.04 \pm 1.23$ & $2.69 \pm 1.59$ \\
\hline Volume $(\mathrm{mL})$ & & $47.91 \pm 20.86$ & & $174.41 \pm 59.36$ \\
\hline Diameter of the pouchoutlet $(\mathrm{cm})$ & $3.91 \pm 0.71$ & $2.16 \pm 0.67$ & & \\
\hline
\end{tabular}

UE: upper endoscopy; 3D-CT: three-dimensional computed tomography volumetry; RYGB: roux-en-Y gastric bypass; SG: sleeve gastrectomy
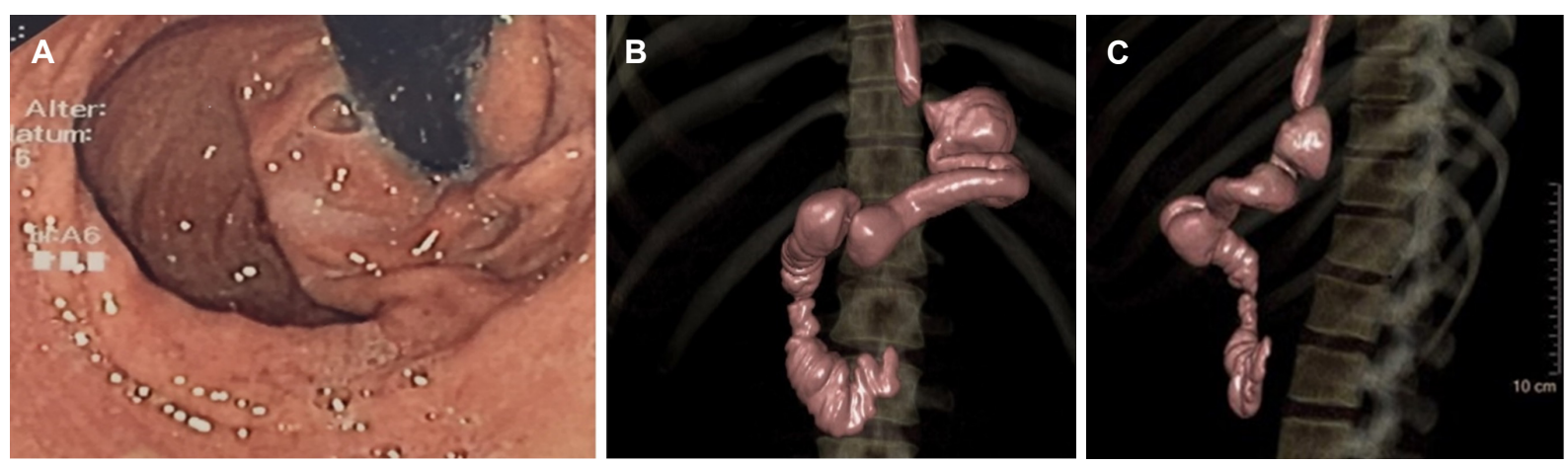

Figure 1. Patient after sleeve gastrectomy with remnant part of fundus, which is herniated to the mediastinum. The sleeve itself is twisted: Endoscopic and 3D-CT view

\section{Pouch-outlet measurement}

Another significant difference was found when comparing the diameter measure of the pouch-outlet in patients with RYGB. With 3D-CT, the mean diameter was $2.16 \pm 0.67 \mathrm{~cm} v s .3 .91 \pm 0.71 \mathrm{~cm}$ with endoscopic measurement $(P<0.001)$ [Table 2].

\section{Clinical cases as visual exemplification of the results \\ Case 1}

A patient after SG with a remnant fundus, which herniated secondarily to a para-oesophageal position. After an odyssey of diagnosis and therapy attempts, the patient was referred in malnourished condition and with recurrent insatiable vomiting and regurgitation. The endoscopic passage was possible without any problems; several external UGIs and even CT scans could not give a decisive clue [Figure 1A-C].

Thus, the indication for examination was the detective assessment of possible underlying anatomical peculiarities. UE already showed the paraesophageal herniation, but could not demonstrate the directly subdiaphragmatic located first bend of the S-shaped kinking. Imaged by $3 \mathrm{D}-\mathrm{CT}$, the adjunctive and crucial anatomical details were firstly a SG double-twist, beginning shortly beyond the diaphragm and secondly the accurate position and tightness of the cardia in relation to the herniation, both exiguous details that were missed during UE and previous external UGIs. According those findings, immediate adhaesiolysis, rest-fundus resection and conversion to RYGB was scheduled after two years of complaints.

\section{Case 2}

A patient after SG with a subtotal stenosis at the angulus fold, which was not easily passable during UE. UGI had shown the very tight stenosis, but only $3 \mathrm{D}$-CT revealed the enormous extent of dilatation of the antrum. The treatment algorithm would have primarily indicated an attempt of dilation. This was dispensed not only because of the tightness of the stenosis, but especially because of the enormous dilatation of the antrum, which needed surgical re-resection [Figure $2 \mathrm{~A}$ and $\mathrm{B}$ ].

The patient underwent direct conversion to RYGB. 

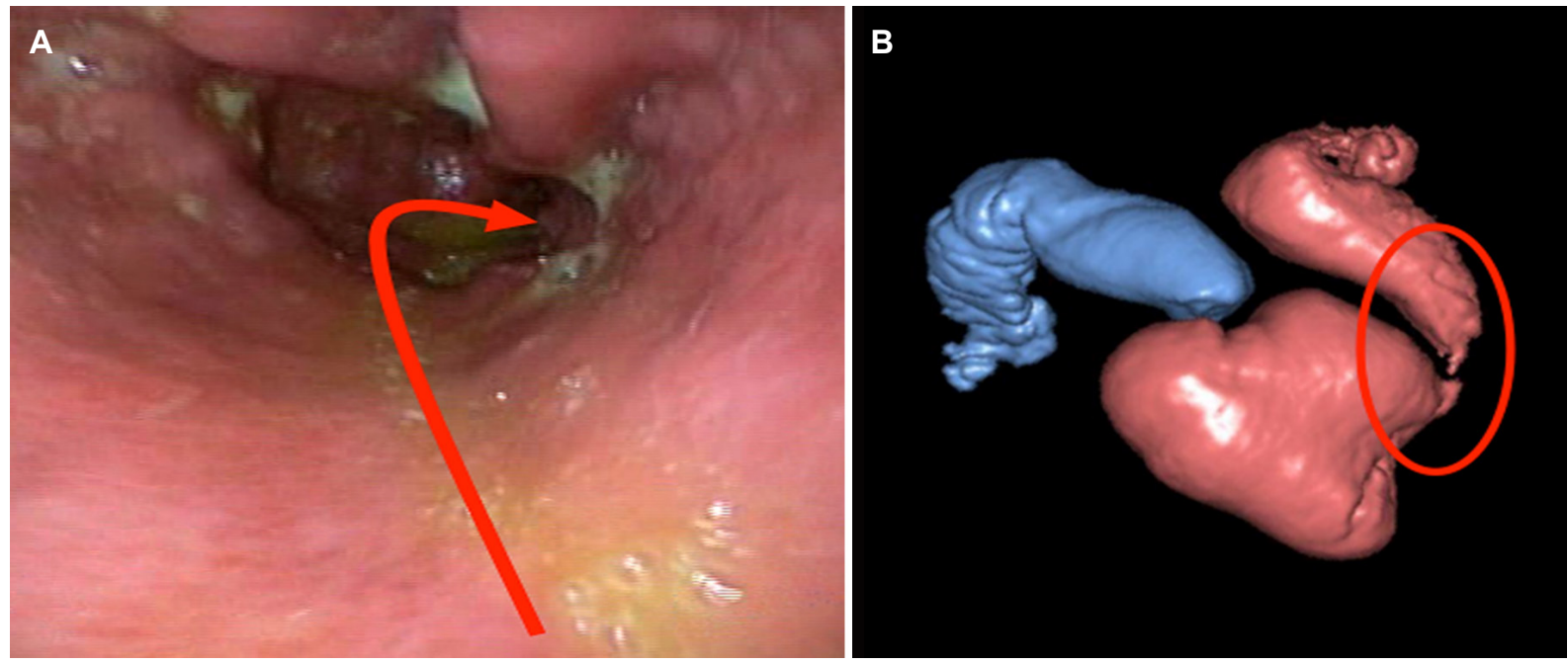

Figure 2. Patient after sleeve gastrectomy with a subtotal stenosis at the angulus fold. Endoscopical and 3D-CT view
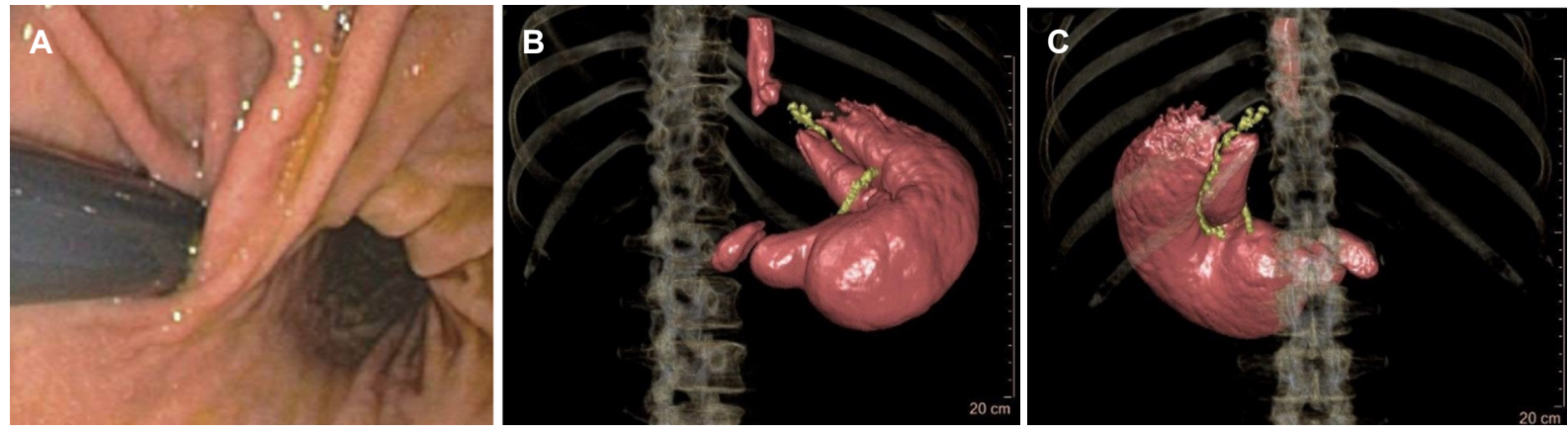

Figure 3. Patient after vertical banded gastroplasty. Endoscopical and 3D-CT view

Case 3

A patient after VBG. The indication for examination was weight regain and non-specific pain in the upper abdomen. Resection lines for the conversion to RYGB were planned with regard to the 3D-CT, which showed perfectly the positioning of the Silastic ring and the length of the vertical partition staple line [Figure 3A-C].

\section{Cases 4 and 5}

Implants and their anatomical position can be surround-viewed from all angles and sides, due to full $360^{\circ}$ rotatability of the images [Figures 4 and 5].

\section{Case 6}

A patient after RYGB. Fully distended Candy Cane, visible from different angles [Figure 6A and B].

\section{DISCUSSION}

Besides the very detailed anatomical pictures, as shown above, which are invaluable as indication and surgery planning guidance in complex revisional surgery, by far the most convincing advantage of 3D-CT is the additional possibility of volumetry.

Weight regain is of special concern in bariatric patients and effective therapy necessitates an objective measurement of gastric volume. Concordantly, our results and the recent results of Hanssen et al ${ }^{[9]}$ clearly 


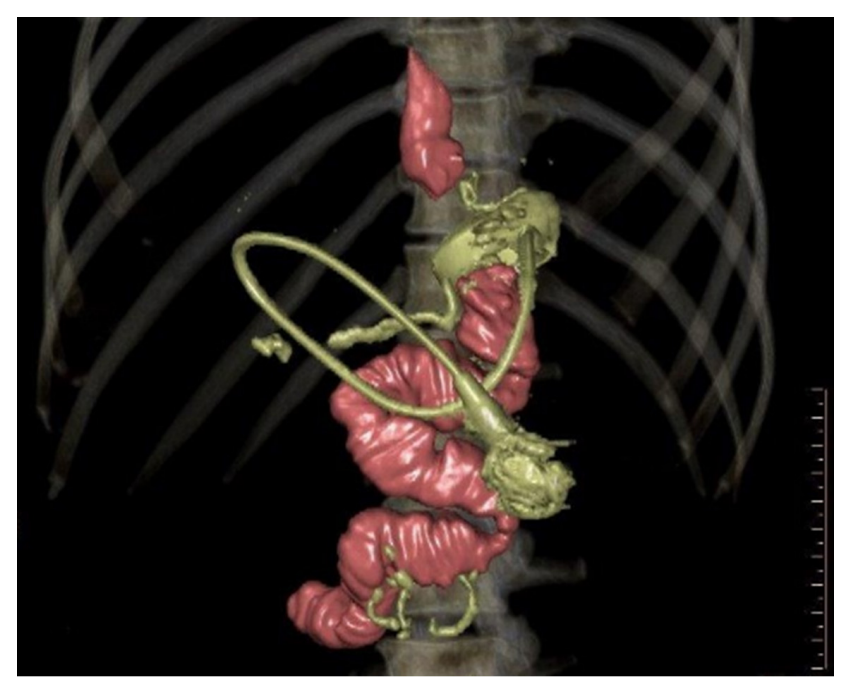

Figure 4. Banded bypass

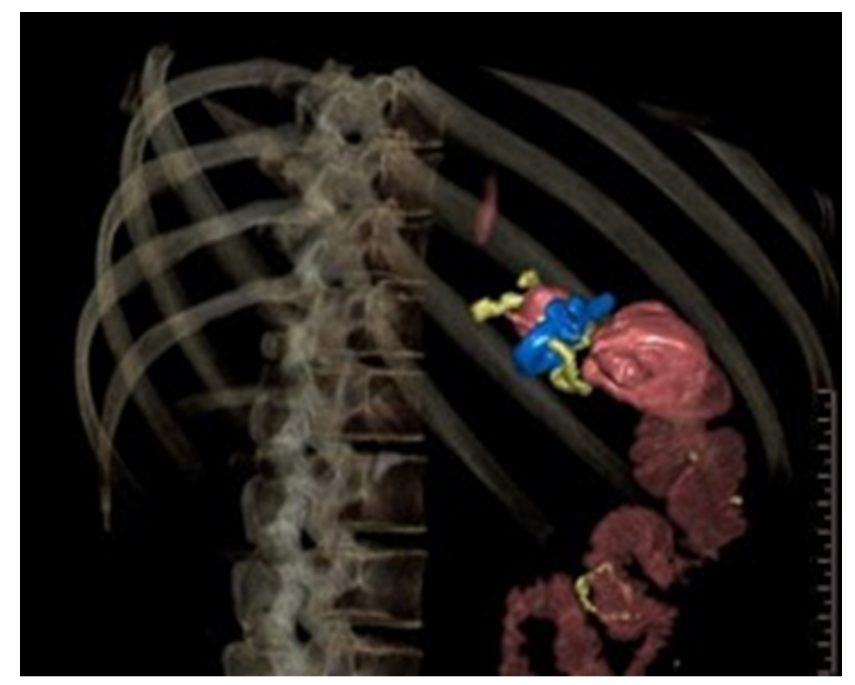

Figure 5. Roux-en-Y gastric bypass with minimiser

demonstrate that SG volume plays a decisive role in weight management (initial weight loss, weight loss failure and possible weight regain). Weight regain is the most feared concern not only in bariatric patients. Actually, SG is globally the most applied bariatric procedure and weight regain occurs frequently after this procedure. 3D volumetry might be a new focus of diagnostic interest for two reasons. Firstly, it shows a rotatable $3 \mathrm{D}$ model of the stomach and its attached structures, even under the most difficult anatomical conditions. In addition, it allows a precise and highly reproducible evaluation of volume alterations of the stomach.

$3 \mathrm{D}-\mathrm{CT}$ is the only accurate diagnostic option available for these purposes. Different bariatric procedures and different surgical techniques lead to varying outcomes, which present challenges pertaining to the evaluation of post-surgical volume and gastro-intestinal (GI) anatomy, especially in regard to the comparability of former, contemporary and future examinations. For this purpose, a protocol should be followed that provides standardised procedural principles for all examinations, thus granting comparability of the results. A diagnostic algorithm is shown in Figure 7. 


\section{A}

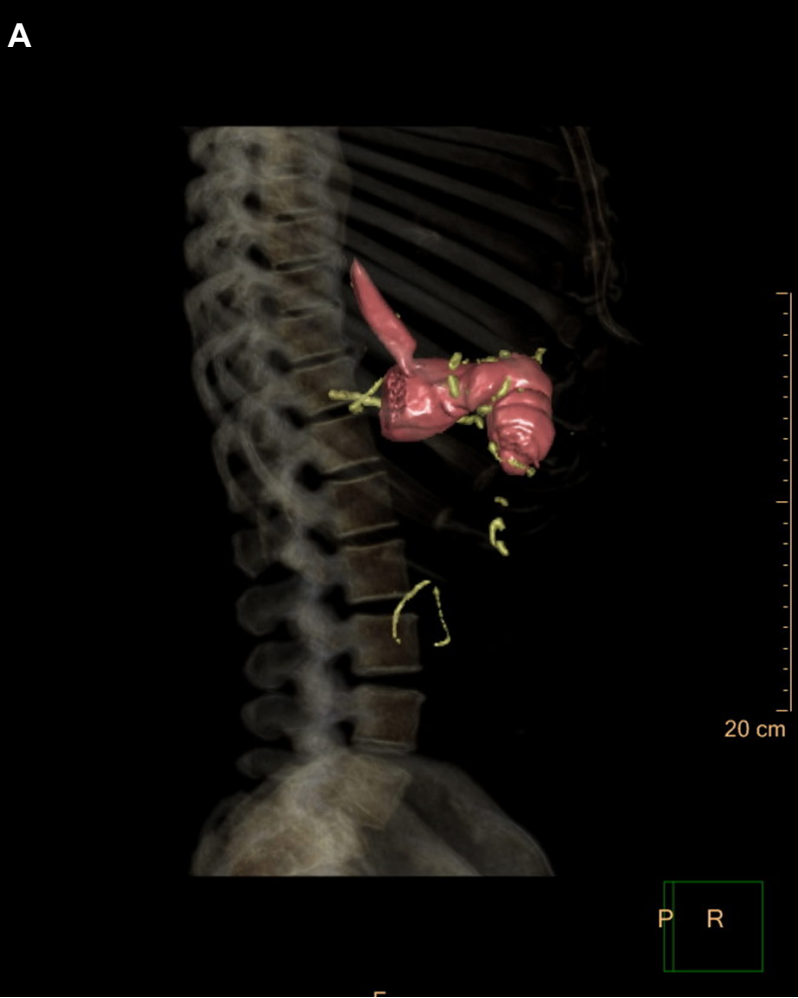

\section{B}

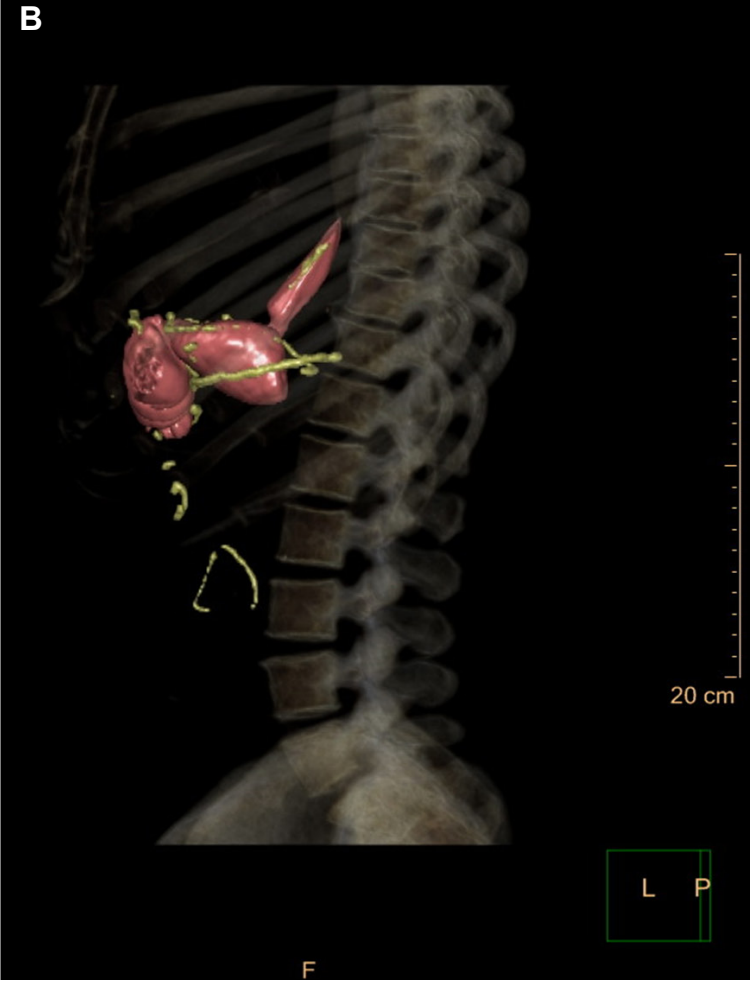

Figure 6. Patient after roux-en-Y gastric bypass. 3D-CT shows the fully tistended candy cane in a $360^{\circ}$ view

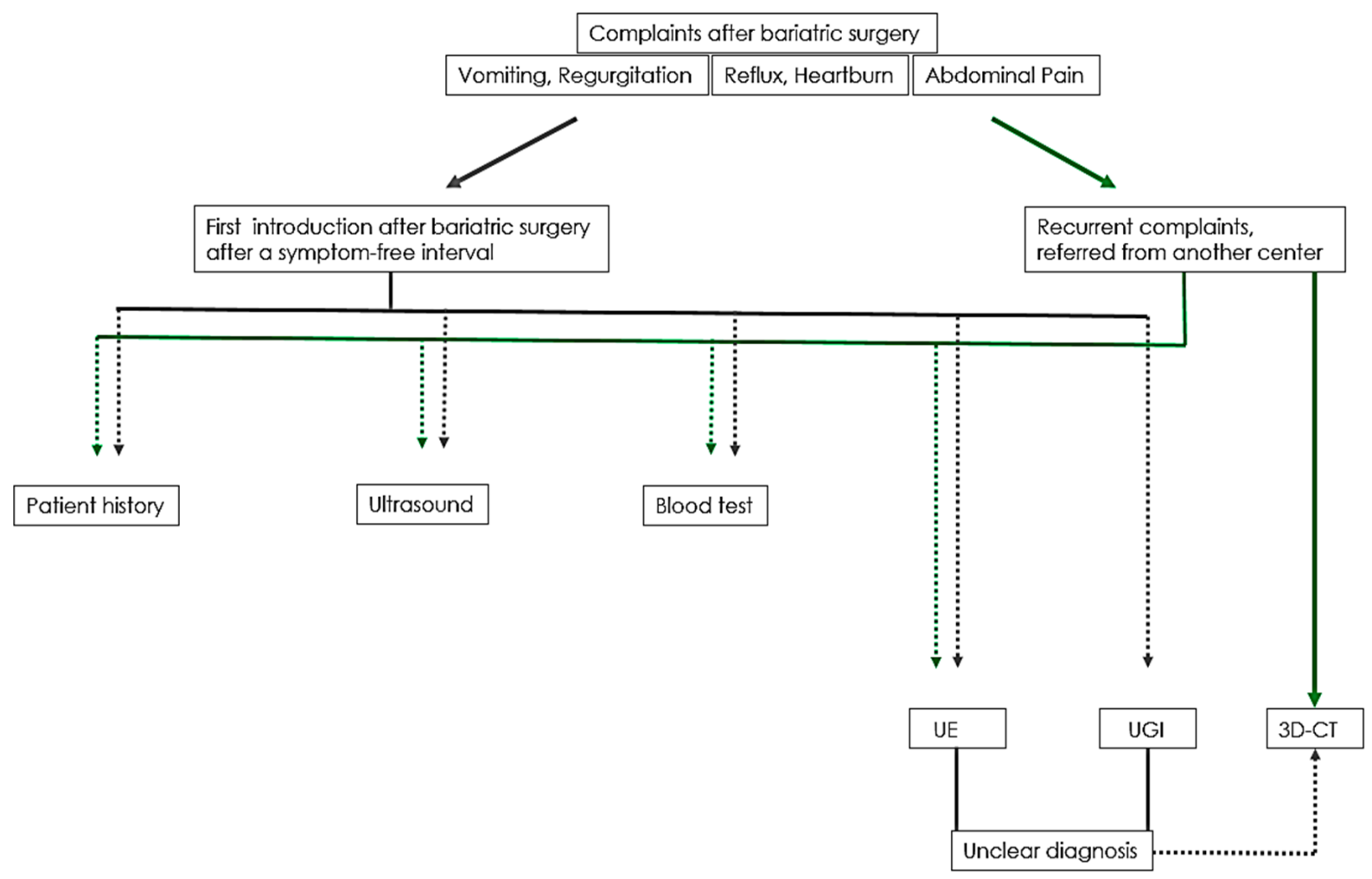

Figure 7. Diagnostic algorithm 
It remains undisputed that, in early perioperative complication management, with special regard to the detection of leaks or stenosis, UGI is the first choice of diagnostic measures. While UE serves as a useful routine examination in patients presenting with upper GI symptoms, 3D-CT allows additionally a more detailed evaluation of post-procedural gastric anatomy and its adjacent structures, enabling easier detection and differentiation of longer-term complications such as sleeve dilatation or thoracic migration ${ }^{[10-14]}$. In sleeve dilatation, a tight sleeve diameter at the angulus fold may cause dysphagia, regurgitation and vomiting after food intake comparable to the symptoms of a hiatal hernia with tight cardia. Thus, thoracic migration is less frequently associated with pure oesophageal reflux symptoms and heartburn. Functional SG stenosis may result in pre-stenotic dilatation of the proximal part of the sleeve. In both entities, 3DCT imaging is a very useful adjunctive diagnostic tool. It shows the functional anatomy that a highly experienced bariatric endoscopist also might be able to notice, but 3D-CT represents the anatomy as examiner-independent, objective imaging.

A further distinct advantage of 3D-CT is the clear depiction of implanted devices (e.g., bands), and their precise anatomical position, which is not possible with UGI due to the lack of tissue extension during the examination.

Additionally, in this study, we evaluated and compared the results of UE with those of 3D-CT for the measurement of the pouch outlet. In contrast, $3 \mathrm{D}-\mathrm{CT}$ is not the diagnostic tool of choice for that purpose.

In almost all cases, the diameter of the Pouch outlet during 3D-CT appeared smaller than was indicated by direct measurement during endoscopy $(P<0.001)$. This may result from the different extension pressures applied during the respective examinations: whereas, in $3 \mathrm{D}-\mathrm{CT}$, the foaming effervescent powder creates enough pressure to gently distend the gastric wall, direct air inflation via endoscope, positioned directly above the anastomosis, causes considerably greater distention. Remarkably, therefore, the pouch outlet after RYGB was significantly underestimated in the examination with 3D-CT.

This is of particular significance in cases of dumping syndrome, a known long-term complication of RYGB, which is often related to an enlarged pouch outlet. For planned outlet reduction procedures in these patients, UE remains the diagnostic tool of choice ${ }^{[15]}$.

From an economical point of view, the costs of 3D-CT are only slightly higher than those of UGI, with current costs of 162.50 euro vs. 225 euro, as calculated by the state health insurance point system in Germany. An additional contrast to the effervescent powder is not necessary for the examination.

3D-CT images reveal three-dimensional information, which is unattainable by alternative examination methods, and allows precise location of the anatomical structures of the upper GI tract. While shape and volume measurements of SG may be repeatedly assessed using this method, the optimal volume of SG or pouch in RYGB remains as yet undefined, but Hanssen et al. ${ }^{[0]}$ recently showed the benefit of a volume $\leq 100 \mathrm{~mL}^{[10]}$. The patients in our SG group had an average volume of $174.41 \pm 59.36 \mathrm{~mL}$ at a reported rate of weight regain of $78.20 \%$. At least this seems to prove that a volume of $174.41 \mathrm{~mL}$ is too large to maintain the gastric restriction and thus leads to a loss of satiety.

3D-CT scan offers a superior technique for the evaluation of volumetric questions, whereas two-dimensional measurements, such as the objectively verifiable diameter of an anastomosis or stenosis, are obviously better assessed by direct measurement with endoscopy.

3D-CT examination requires a bariatrically trained radiology team with a standardised protocol for best results, as described above. 
This study is limited by the retrospective nature of the data analysis. In addition, the data were collected in a single-centre study, although $3 \mathrm{D}-\mathrm{CT}$ is a well-established diagnostic tool in this high-volume certified centre of excellence for obesity and metabolic surgery.

To conclude, 3D-CT is quick, easy-to-perform and facilitates identification of the post-surgical gastric anatomy. It represents a valuable additional diagnostic tool in post-bariatric patients with post-procedural complications, since UE and UGI might miss the three-dimensional post-bariatric anatomy. 3D-CT might be an important preoperative tool prior to revisional surgery and an ideal diagnostic complement in patients with post-surgical complications following obesity surgery.

\section{DECLARATIONS}

\section{Authors' contributions}

Designed the article: Stier C, Chiappetta S

Calculated the statistics and proofed the concept: Parmar C, Koschker AC, Stier R

Operating radiologist: Bokhari M

\section{Availability of data and materials}

Data route from the results of routine examinations of patients. Anonymized data sheet is available from the authors (Stier C).

\section{Financial support and sponsorship}

None.

\section{Conflicts of interest}

All authors declared that there are no conflicts of interest.

\section{Ethical approval and consent to participate}

All procedures performed in this study involving human participants were in accordance with the ethical standards of the institutional and/or national research committee and with the 1964 Helsinki Declaration and its later amendments or comparable ethical standards.

\section{Consent for publication}

Not applicable.

\section{Copyright}

(c) The Author(s) 2020.

\section{REFERENCES}

1. Sjöström L, Lindroos AK, Peltonen M, Torgerson J, Bouchard C, et al.; Swedish Obese Subjects Study Scientific Group. Lifestyle, diabetes, and cardio- vascular risk factors 10 years after bariatric surgery. N Engl J Med 2004;351:2683-93.

2. Schauer PR, Bhatt DL, Kirwan JP, Wolski K, Aminian A, et al. Bariatric surgery versus intensive medical therapy for diabetes - 5-year outcomes. N Engl J Med 2017;376:641-51.

3. Mingrone G, Panunzi S, De Gaetano A, Guidone C, Iaconelli A, et al. Bariatric-metabolic surgery versus conventional medical treatment in obese patients with type 2 diabetes: 5 year follow-up of an open-label, single-centre, randomised controlled trial. Lancet 2015;386:964-73.

4. Healy P, Clarke C, Reynolds I, Arumugasamy M, McNamara D. Complications of bariatric surgery--What the general surgeon needs to know. Surgeon 2016;14:91-8.

5. Livingston EH. Complications of bariatric surgery. Surg Clin North Am 2005;85:853-68.

6. Abd Ellatif ME, Alfalah H, Asker WA, El Nakeeb AE, Magdy A, et al. Place of upper endoscopy before and after bariatric surgery: a multicenter experience with 3219 patients. World J Gastrointest Endosc 2016;8:409-17.

7. Brethauer SA, Nfonsam V, Sherman V, Udomsawaengsup S, Schauer PR, et al. Endoscopy and upper gastrointestinal contrast studies 
are complementary in evaluation of weight regain after bariatric surgery. Surg Obes Relat Dis 2006;2:643-8.

8. Nagpal P, Prakash A, Pradhan G, Vidholia A, Nagpal N, et al. MDCT imaging of the stomach: advances and applications. Br J Radiol 2017;90:20160412.

9. Hanssen A, Plotnikov S, Acosta G, Nuñez JT, Haddad J, et al. 3D volumetry and its correlation between postoperative gastric volume and excess weight loss after sleeve gastrectomy. Obes Surg 2018;28:775-80.

10. Robert M, Pechoux A, Marion D, Laville M, Gouillat C, et al. Relevance of Roux-en-Y gastric bypass volumetry using 3-dimensional gastric computed tomography with gas to predict weight loss at 1 year. Surg Obes Relat Dis 2015;11:26-31.

11. Blanchet MC, Mesmann C, Yanes M, Lepage S, Marion D, et al. 3D gastric computed tomography as a new imaging in patients with failure or complication after bariatric surgery. Obes Surg 2010;20:1727-33.

12. Kawamoto S, Fishman EK. Adjustable laparoscopic gastric banding: Demonstrated on mutlidetector computed tomography with multiplanar reformation and 3-dimensional imaging. J Comput Assist Tomogr 2009;33:288-90.

13. Karcz WK, Kuesters S, Marjanovic G, Suesslin D, Kotter E, et al. 3D-MSCT gastric pouch volumetry in bariatric surgery - preliminary clinical results. Obes Surg 2009;19:508-16.

14. Baumann T, Grueneberger J, Pache G, Kuesters S, Marjanovic G, et al. Three-dimensional stomach analysis with computed tomography after laparoscopic sleeve gastrectomy: sleeve dilation and thoracic migration. Surg Endosc 2011;25:2323-9.

15. Stier C, Chiappetta S. Endoluminal revision (OverStitch (TM), apollo endosurgery) of the dilated gastroenterostomy in patients with late dumping syndrome after proximal roux-en-Y gastric bypass. Obes Surg 2016;26:1978-84. 